\title{
12. MODELS FOR COMPACT PULSING X-RAY SOURCES
}

\author{
JEREMIAH P. OSTRIKER and KRIS DAVIDSON \\ Princeton University Observatory, Princeton, N.J., U.S.A.
}

\begin{abstract}
Cen X-3 is probably a neutron star, releasing the infall energy of accreted matter. Sufficient material for accretion will be provided by a conventional stellar wind from its more massive companion star. That star is not likely to rotate synchronously; therefore a 'Roche lobe' analysis of the eclipses is not valid. A 'tidal lobe' analysis allows the neutron star to have a mass of the order of one solar mass. Overflow of the 'Roche lobe' is neither necessary as a source of mass nor probable in view of the observed stellar line widths of the two identified X-ray companions.

The mass flow onto the condensed star is very small in all cases. It is limited, for an object of $m$ solar masses by the Eddington Limiting Luminosity to $\dot{M}_{\mathrm{ac}}<10^{-7.4} m\left(M \odot \mathrm{yr}^{-1}\right)$, which limit applies even if the accreting object contacts or traverses its companion star.

The observed $4.84 \mathrm{~s}$ rotation period of the Cen X-3 neutron star is very simply explained as the critical value where a centrifugal barrier regulates the rate of infall to the surface. The X-ray spectrum is understood as blackbody radiation coming from a well-defined area near each magnetic pole of the neutron star.
\end{abstract}

\section{General Remarks: Accretion as a Source of Energy}

We restrict attention to those X-ray sources which show significant variations on time scales of one second or less and which do not indicate pulsed radio emission. Since rotation, pulsation, and orbital periods are usually limited to $P \geqslant(G \varrho)^{-1 / 2}$, we require objects with mean densities greater than $10^{7} \mathrm{~g} \mathrm{~cm}^{-3}$ - which, by the usual arguments, restricts consideration to degenerate ('white') dwarfs, neutron stars, and black holes.

We focus primarily on the class of X-ray variables whose prototypes are the sources Cen X-3 and Her X-1; these emit fairly regular X-ray pulses with periods of 4.842 and $1.238 \mathrm{~s}$, show regular phase variations, and have apparent eclipses with periods of 2.087 and $1.700 \mathrm{~d}$ (Schreier et al., 1972; Tananbaum et al., 1972). According to convincing interpretations of the observations by the above authors, both objects are in nearly circular orbits around stars (which remain unidentified at this time); the mass functions in the two cases are $15 M_{\odot}$ and $0.85 M_{\odot}$. Estimates for the distances and hence the X-ray luminosities of the variable sources are highly uncertain, but statistical considerations (Salpeter, 1972) and absorption of the lower-energy X-rays seem to indicate typical distances of $1-5 \mathrm{kpc}$ and luminosities of $10^{36}-10^{38} \mathrm{erg} \mathrm{s}^{-1}$.

If these guesses are even approximately correct, the periodic sources cannot be degenerate dwarfs: for although very short period regular variations due to either pulsation or rotation are possible in massive, rapidly rotating cold dwarfs (Ostriker and Tassoul, 1968; Gribben, 1971), it is easy to show that the high surface temperature required for an X-ray source would produce a distended atmosphere which would lengthen the minimum rotation and pulsation periods to values in excess of one second. Salpeter (1972) has given other arguments against the pulsating white dwarf hypotheses.

The periodic sources may be neutron stars or black holes with modulation due to rotation or to orbital motions; but orbital motions would lead to period changes 
(Ostriker, 1968) in excess of those allowed by observations, and non-axisymmetric black holes can have only a transient existence (if any). Thus we are led to rotating neutron stars by almost the same chain of arguments which indicated these hypothetical objects as the bases of the radio 'pulsars'.

In a brief assay of the statistical problem, Gott et al., (1970) estimated that perhaps $\frac{1}{15}$ of all pulsars should be found in binary systems. This was probably a severe underestimate, since they neglected the likelihood that mass transfer in a close binary system causes the first supernova explosion to occur in the less massive star. Consequently, more than half of the mass is typically retained in the system, and then, as pointed out by van den Heuvel and Heise (1972), the newly created neutron star will usually remain bound to its more massive companion. Yet none of the 60 -odd known radio pulsars seems to be a member of a binary system. Evidently neutron stars in binary systems must do something else - and they may become the periodic X-ray systems.

The X-ray luminosities in these systems cannot, however, derive from rotation, as is thought to be the case in the Crab Nebula. If rotation were the energy source, then, since $L_{x} \sim 10^{37} \mathrm{erg} \mathrm{s}^{-1}$ for both the Crab Nebula and an X-ray pulsar, we must have

$$
(I \Omega S)_{X} \approx(I \Omega S)_{\text {Crab pulsar }}
$$

Therefore, the characteristic time for period increase is

$$
\begin{aligned}
\tau_{X} \equiv(\Omega / \Omega)_{X} & \approx(\Omega / \Omega)_{\mathrm{Crab}}\left(\Omega_{X} / \Omega_{\mathrm{Crab}}\right)^{2} \\
& \approx\left(3 \times 10^{-5}\right) \tau_{\mathrm{Crab}} \\
& \approx 0.06 \mathrm{yr},
\end{aligned}
$$

which is orders of magnitude shorter than the observationally permitted rate of period change. In other words, the observed energy output is far more than that available from a relatively slowly rotating neutron star. Internal stored thermal energy is likewise probably insufficient, though not by so large a margin. After considering and discarding other potential sources of energy, we are left with accretion of matter unto the neutron star's surface as the only plausible energy source (for an excellent review of the accretion phenomenon see Zel'dovich and Novikov, 1971). The total energy released per unit time is the rate of proton accretion times $\mathrm{d} E(B) / \mathrm{d} B$, where $E(B)$ is the neutron binding energy as a function of baryon number. ${ }^{*} \mathrm{~A}$ one-solar mass neutron star requires an accretion rate of about $10^{-9} M_{\odot} \mathrm{yr}^{-1}=10^{16.8} \mathrm{~g} \mathrm{~s}^{-1}$ to release a total of $10^{37} \mathrm{erg} \mathrm{s}^{-1}$.

\section{The Source of the Mass Flow}

The required mass flux, while small, is more than can plausibly be expected to accrete from the interstellar medium (Ostriker et al., 1970). However, long before the dis-

* Many authors have incorrectly used the surface potential rather than $\mathrm{d} E(B) / \mathrm{d} B$, neglecting the additional energy liberated as the neutron star adjusts to its increased mass, as well as the correction for the 'redshift' of the escaping energy in the observer's frame. However, the general-readjustment energy is likely to appear in a different form from the initial-infall energy. 
covery of the objects considered here, Zel'dovich and Novikov had suggested (1964) that condensed stars would be most easily found as X-ray sources accreting matter from binary companions. Material can become available for accretion by at least two processes: first, many types of single stars lose mass at significant rates; in particular, early-type stars are observed to be ejecting mass at rates typically exceeding $10^{-7} M_{\odot} \mathrm{yr}^{-1}$, with velocities of the order of $10^{3} \mathrm{~km} \mathrm{~s}^{-1}$. This phenomenon is easily discerned in violet-shifted emission lines of CIV and SIV which appear in the rocket UV spectra by Morton (1967, 1969) and Carruthers (1968), and may occasionally be seen in ground-based spectra (Beals, 1951). Such a star, in a binary system, would provide a source of mass for accretion by its companion. Alternatively, in a very close binary, tidal action may assist in the removal of material from the surface of a star which would not otherwise be losing matter. In an extreme case, if the stellar spin rate is equal to the orbital revolution rate, a star might thus 'overflow its Roche lobe'. We note, however, that there is in fact no reason to suppose that the star must rotate synchronously.

In this connection, consider the upper limit which has recently been suggested by several authors (cf. Wilson, 1972; Leach and Ruffini, 1972; van den Heuvel and Heise, 1972) for the mass of the accreting object in Cen X-2. The chain of argument has three links:

(1) The duration of the X-ray eclipse determines a function of the ratio $a / R_{*}=$ (binary separation)/(radius of the large eclipsing star) and of the orbital inclination $i$. (The inclination $i$ is $90^{\circ}$ if the observer is in the plane of the orbit.)

(2) The sinusoidal Doppler variation of the $4.84 \mathrm{~s}$ period, as the X-ray source orbits around the large star, determines the 'mass function', (M.F.) $=M_{*}^{3} \sin ^{3} i /\left(m_{x}+M_{*}\right)^{2}$, where $m_{x}$ is the mass of the X-ray source and $M_{*}$ is the mass of the larger companion.

(3) The requirement that $m_{x}$ must not remove mass from $M_{*}$ too quickly by tidal forces, under the assumption that $M_{*}$ rotates synchronously with the orbital period-i.e., the larger star must not be larger than its Roche lobe - determines the maximum acceptable value of the mass ratio $m_{x} / M_{*}$ as a function of $a / R_{*}$. This, with the mass function, implies a maximum possible $m_{x}$ for a given inclination $i$.

The lower curve in Figure 1 shows $\theta_{0}$, the angle in the orbital plane subtended by the larger Roche lobe as seen from $m_{x}$, as a function of $M_{*} / m_{x}$. The upper curve shows $\theta_{0}$ for a tidal lobe, in the case where the larger star is not rotating and the Roche potentials have little significance (see Jeans, 1919 and 1928). For a given inclination $i$, $\theta_{0}$ may be estimated from the apparent eclipse angle $\theta$, and then the minimum value of $M_{*} / m_{x}$ may be found from Figure 1 . It is clear that larger values of $m_{x}$ are permitted in the non-rotating case; and as a rule, early-type stars in close binary systems do not rotate synchronously (van den Heuvel, 1970), probably because tidal forces take longer to operate than the stellar lifetimes. We also note that the two known or probable companions of X-ray sources which have been spectroscopically studied, HDE 226868 (Bolton, 1971; Webster and Murdin, 1972) and HD 77581 (Hiltner et al., 1972), do not show lines wide enough to indicate synchronous rotation. Therefore, slow rotation seems to be appropriate. 


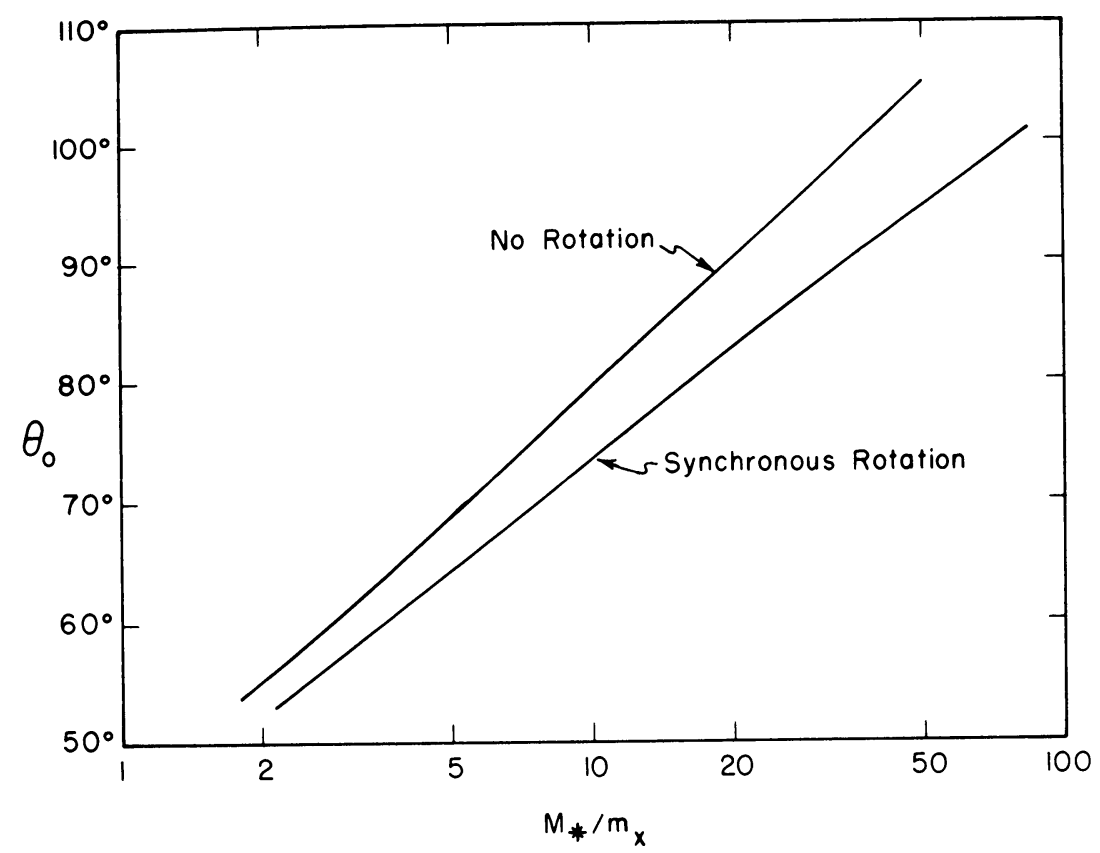

Fig. 1. Angle in orbital plane eclipsed by larger tidal lobe, as seen from smaller companion, for various mass ratios.

According to Schreier et al. (1972), Cen X-3 is totally eclipsed for $0.488 \mathrm{~d}$ during the orbital period of $2.087 \mathrm{~d}$, indicating $\theta \approx 84^{\circ}$. Following Wilson (1972), one might increase this to about $96^{\circ}$ by including the transition periods at the beginning and end of each eclipse, but these are evidently caused by an extended atmosphere, which may be moving dynamically outside the critical tidal lobe. Therefore, adopting the smaller value and the observed mass function, M.F. $=15 M_{\odot}$ (Schreier et al., 1972), one finds

TABLE I

Maximum masses in Cen X-3

\begin{tabular}{lrcccl}
\hline$i$ & \multicolumn{1}{c}{$\theta_{0}$} & $R^{*} / r$ & $d_{\text {crit }} / r$ & $M^{*} / M_{\odot}$ & $m_{x} / M_{\odot}$ \\
\hline $90^{\circ}$ & $84^{\circ} .5$ & 0.67 & 0.21 & 17.2 & 1.24 \\
$80^{\circ}$ & $86^{\circ} .4$ & 0.68 & 0.20 & 17.8 & 1.13 \\
$70^{\circ}$ & $91^{\circ} .9$ & 0.72 & 0.17 & 19.8 & 0.90 \\
$60^{\circ}$ & $100^{\circ} .3$ & 0.77 & 0.14 & 24.3 & 0.65 \\
$50^{\circ}$ & $110^{\circ} .9$ & 0.82 & 0.10 & 34.2 & 0.43 \\
\hline
\end{tabular}

$i=$ inclination of orbit;

$\theta_{0}=$ angle eclipsed, in plane of orbit;

$r=$ separation of the two components;

$R^{*}=$ average radius of larger component;

$d_{\text {crit }}=$ distance from X-ray source to the cusp of the larger star's tidal lobe;

$m_{x}=$ maximum mass of X-ray source;

$M^{*}=$ maximum mass of larger component. 
the maximum acceptable masses listed in Table I, for various inclinations. Unless the inclination is small (in which case $M_{*}$ would be surprisingly large), $m_{x}$ may have a very conventional neutron-star mass. In the case of Her X-1, where the mass function is only $0.85 M_{\odot}$ and $\theta$ is $60^{\circ}$ or less (Tananbaum et al., 1972), plausible values (e.g., $M_{*} \sim 2 M_{\odot}, m_{x} \sim 1 M_{\odot}$ ) are possible if $i$ is not much less than $90^{\circ}$.

While not excluding the possibility that tidal forces may drive some mass outflow, here we shall investigate models in which the material to be accreted appears in the form of a stellar wind.

\section{Accretion Rate and Luminosity}

Consider a neutron star of mass $m_{x}$, travelling at velocity $v_{\text {rel }}$ through a gas of density $\varrho$. if $v_{\text {rel }}$ is much larger than the sound speed, the gas flows in the manner described by Bondi and Hoyle (1944). If some gas initially flows within a cylinder of radius $a_{\mathrm{accr}}$ $\approx 2 \mathrm{G} m_{x} / v_{\text {rel }}^{2}$, then it loses enough energy in passing through the tail shock to fall back towards the star (see Figure 2). The complicated flow near the rotating magnetic neutron star will be discussed in the next section. The accretion rate is then

$$
\dot{M}_{\mathrm{accr}}=\pi \zeta a_{\mathrm{accr}}^{2} v_{\mathrm{rel}} \varrho,
$$

where $\zeta$, which is less than unity, is a factor correcting for the repulsion of infalling material by radiation pressure. Radiation pressure, acting on electrons, provides

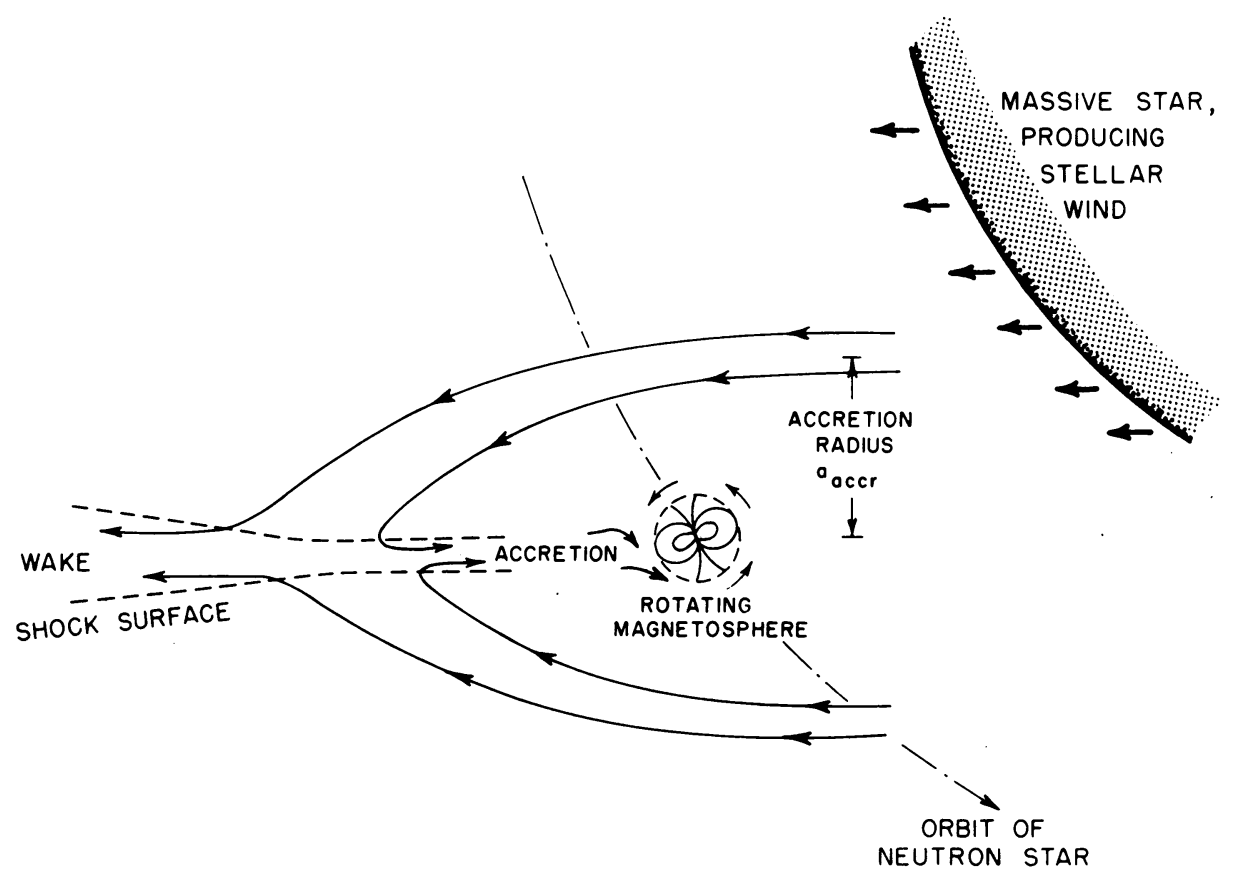

Fig. 2. Streamlines of stellar-wind material, in frame of an accreting neutron star. Relative dimensions are not to scale. 
a $1 / r^{2}$ repulsive force which effectively reduces the gravity of the central object:

$$
\zeta=\left[1-10^{-4.8}(1+X)\left(L_{x} / L_{\odot}\right) /\left(m_{x} / M_{\odot}\right)\right]^{2},
$$

where $X$ is the fractional abundance of hydrogen in the gas and $L_{x}$ is the luminosity of the accreting object (Eddington, 1926). Equation (2), of course, is valid only if the radiation is isotropic, the surrounding medium optically thin, and the radiation $L_{x}$ not so hard that the Thomson cross-section is appreciably reduced $(h v<0.5 \mathrm{MeV})$.

The total luminosity resulting from accretion is, as mentioned earlier,

$$
L_{x}=\left(\dot{M}_{\mathrm{accr}} / m_{\mathrm{H}}\right)[\mathrm{d} E(B) / \mathrm{d} B] \approx\left(10^{46} \mathrm{erg} \mathrm{s}^{-1}\right)\left(\dot{M}_{\mathrm{accr}} / M_{\odot} \mathrm{yr}^{-1}\right),
$$

where $m_{\mathrm{H}}$ is atomic mass unit. We see from Equations (1), (2), and (3) that radiation pressure acts to limit the luminosity and accretion rate:

$$
\begin{aligned}
& L_{x}<L_{\text {crit }}=\left(10^{4.8} L_{\odot}\right)\left(m_{x} / M_{\odot}\right) /(1+X), \\
& \dot{M}_{\text {accr }}<\dot{M}_{\text {crit }}=\left(10^{18} \mathrm{~g} \mathrm{~s}^{-1}\right)\left(m_{x} / M_{\odot}\right) \\
&=\left(10^{-7.4} M_{\odot} \mathrm{yr}^{-1}\right)\left(m_{x} / M_{\odot}\right),
\end{aligned}
$$

which is probably low enough to preclude significant change of the mass $m_{x}$ during the lifetime of a massive stellar system. Note that the limiting process makes the luminosity depend only very weakly on the details of the accretion mechanism as the limiting luminosity is approached.

We now consider the nature of the stellar wind. According to Lucy and Solomon (1970), the mass flux $\dot{M}_{w}$ and general characteristics of the flow are established by the time the sonic point is reached. At this point, which occurs not far $\left(\sim 10^{10.5} \mathrm{~cm}\right)$ from the surface of a giant star, the velocity is small $\left(v_{s} \approx 20 \mathrm{~km} \mathrm{~s}^{-1}\right)$. In the supersonic regime, the flow simulates pressure-free particles moving in an inverse-square repulsive force field. Then,

$$
v_{w}^{2}=v_{s}^{2}+\left(1-R_{s} / r\right) v_{\infty}^{2} \approx\left(1-R_{*} / r\right) v_{\infty}^{2},
$$

where $v_{\infty}$ is the asymptotic flow velocity toward infinity. The second form of Equation (6) is suitable at distances more than about $10^{11} \mathrm{~cm}$ from the stellar surface. The orbital velocity of the neutron star,

$$
v_{x}^{2}=G M_{*} / r=v_{\mathrm{esc}}^{2} R_{*} / 2 r,
$$

must be included to get the wind velocity relative to the neutron star:

$$
v_{\mathrm{rel}}^{2}=v_{x}^{2}+v_{w}^{2}=\left[1-\left(1-v_{\mathrm{esc}}^{2} / 2 v_{\infty}^{2}\right)\left(R_{*} / r\right)\right] v_{\infty}^{2} .
$$

The density in the stellar wind is, from the continuity equation,

$$
\varrho=\frac{\dot{M}_{w}}{4 \pi r^{2} v_{w}}=\frac{\left(\dot{M}_{w} / 4 \pi v_{\infty}\right)}{r^{2}\left(1-R_{*} / r\right)^{1 / 2}}
$$


Combining the above equations gives

$$
\dot{M}_{\mathrm{accr}}=\dot{M}_{w} \frac{4\left(m_{x} / M_{*}\right)^{2} \varepsilon^{2}}{(x-1)^{1 / 2}(x-1+\varepsilon)^{3 / 2}}\left[1-\frac{L_{x}}{L_{\mathrm{crit}}}\right]^{2},
$$

where $\varepsilon=v_{\text {esc }}^{2} / 2 v_{\infty}^{2}$ and $x=r / R_{*}$.

We may now construct a few exemplary quantities to show what Cen X-3 might be like. Suppose that $m_{x}=1 M_{\odot}, M_{*}=17 M_{\odot}, R_{*}=9 \times 10^{11} \mathrm{~cm}$, and $r=1.3 \times 10^{12} \mathrm{~cm}$, consistent with observations and with Table I; then $x=1.4$ and $v_{\text {esc }}=500 \mathrm{~km} \mathrm{~s}^{-1}$. Suppose that $L_{x}=10^{37} \mathrm{erg} \mathrm{s}^{-1}=0.1 \dot{M}_{\mathrm{accr}} c^{2}$ (only part of this may be observable; see Section 4 below); so we require $\dot{M}_{\text {accr }}=10^{17} \mathrm{~g} \mathrm{~s}^{-1}$ while $\zeta=\left(1-L_{x} / L_{\text {crit }}\right)^{2}=0.8$. Then, necessary values of the stellar mass-loss rate $M_{w}$, according to Equation (10), are shown in Table II for several values of $v_{\infty}$. These rates are of the order of $10^{-6}$ $M_{\odot} \mathrm{yr}^{-1}-$ not at all exorbitant, especially if tidal forces assist the ejection of material from the massive star.

TABLE II

Necessary stellar mass-loss rates (If the total X-ray production in

Cen $\mathrm{X}-3$ is $10^{37}$ ergs s$^{-1}$ )

\begin{tabular}{rlll}
$\begin{array}{l}v_{\infty} \\
\left(\mathrm{km} \mathrm{s}^{-1}\right)\end{array}$ & $\varepsilon$ & $\begin{array}{l}M_{w} \\
\left(\mathrm{~g} \mathrm{~s}^{-1}\right)\end{array}$ & $\left(M_{\odot} \mathrm{yr}^{-1}\right)$ \\
\hline 500 & 0.5 & $2.0 \times 10^{19}$ & $3 \times 10^{-7}$ \\
1000 & 0.125 & $1.4 \times 10^{20}$ & $2 \times 10^{-6}$ \\
1500 & 0.056 & $5.5 \times 10^{20}$ & $9 \times 10^{-6}$
\end{tabular}

It is also relevant that, since the $\mathrm{X}$-ray source is rather close to the surface of the larger star, the X-ray flux, incident from above, may perturb the stellar atmosphere in a small region around the sub-neutronstar point. Some of the lower-energy X-rays are likely to be absorbed in or above the photosphere, thereby heating the region and provoking additional mass-ejection.

\section{Behavior of Gas near the Accreting Object}

The accreted gas falls toward the neutron star within a narrow, conical tail shock (see Figure 2) until it reaches a rather small radius. Then, if the neutron star has a dipole-like magnetic field, the infalling material must encounter a region where its pressure is exceeded by magnetic pressure: the magnetosphere (see Figure 3 ). Within this region, matter is constrained to follow the co-rotating field lines. Gas is likely to collect around the boundary of the magnetosphere, whose size is readily estimable.

Denote the radius of the magnetosphere by $R_{m}$; while $R_{0}$ is the radius of the neutron star, $R_{G}=G m_{x} / c^{2}$ is half the Schwarzschild radius of the neutron star, and $R_{c}=c / \Omega$ is the radius of the speed-of-light cylinder where the angular rotation speed is $\Omega$. If $B_{0}$ is the surface magnetic field, then the field at the magnetopause is approximately 


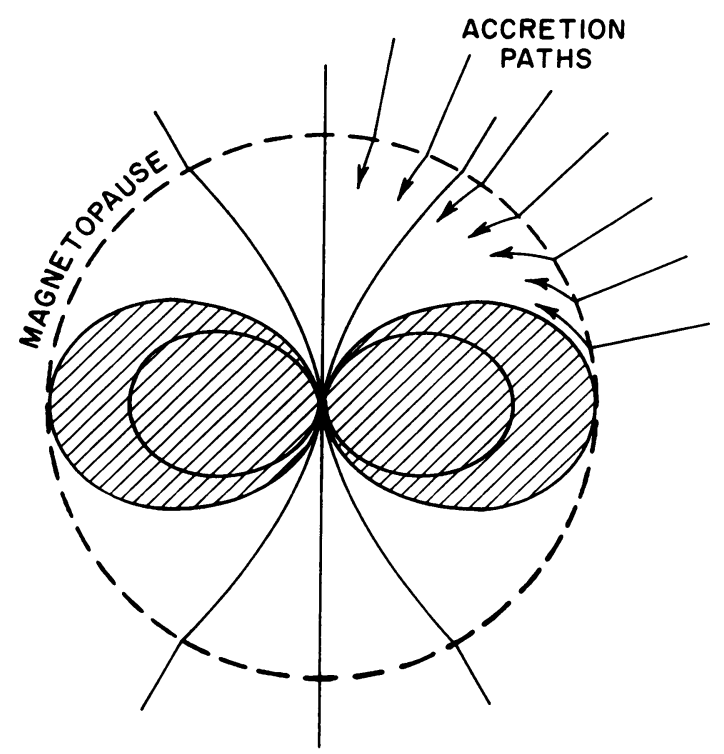

Fig. 3. A simplified dipole magnetosphere around an accreting neutron star.

$B_{m}=B_{0} R_{0}^{3} / R_{m}^{3}$ and the magnetic pressure is $B_{m}^{2} / 8 \pi$. We approximate the gas pressure at $R_{m}$ by a rampressure $\varrho v_{\text {infall }}^{2}$, where $v_{\text {infall }}^{2} \approx R_{G} c^{2} / R_{m}$. Then (ram-pressure) $\approx$ $\approx \dot{M}_{\mathrm{accr}} v_{\mathrm{infall}} / 4 \pi R_{m}^{2}=\left(\dot{M}_{\mathrm{accr}} c / 4 \pi\right) R_{G}^{1 / 2} R_{m}^{-5 / 2}$. Equating magnetic and gas pressures, we find

$$
\begin{aligned}
& R_{m} \approx\left[\frac{B_{0}^{2} R_{0}^{6}}{2 \dot{M}_{\mathrm{accr}} c R_{\mathrm{G}}^{1 / 2}}\right]^{2 / 7}=\left(3 \times 10^{8} \mathrm{~cm}\right)\left(\frac{B_{0}}{10^{12} \mathrm{G}}\right)^{4 / 7}\left(\frac{R_{0}}{10 \mathrm{~km}}\right)^{12 / 7} \times \\
& \times\left(\frac{\dot{M}_{\mathrm{accr}}}{10^{17} \mathrm{~g} \mathrm{~s}^{-1}}\right)^{-2 / 7}\left(\frac{m_{x}}{M_{\odot}}\right)^{-1 / 7}
\end{aligned}
$$

If Cen X-3 has a surface field of the order of $10^{12} \mathrm{G}$ (like the pulsars), and $R_{0} \approx 14 \mathrm{~km}$, we expect $R_{m} \approx 5 \times 10^{8} \mathrm{~cm} \ll R_{c}$.

We now preface the spin-angular momentum problem by noting a suggestive coincidence. The radius of a synchronous orbit, where the gravitational orbital velocity would be in co-rotation with the spinning neutron star, is

$$
\begin{aligned}
R_{\text {sync }} & =R_{\mathrm{G}}^{1 / 3} R_{c}^{2 / 3} \\
& =\left(1.5 \times 10^{8} \mathrm{~cm}\right)\left(m_{x} / M_{\odot}\right)^{1 / 3}(P / 1 \mathrm{~s})^{2 / 3},
\end{aligned}
$$

where $P$ is the period of rotation. For Cen $X-3(P=4.84 \mathrm{~s}), R_{\text {sync }} \approx 4.3 \times 10^{8} \mathrm{~cm}$, which is very close to $R_{m}$. This is suggestive because it means that if Cen $X-3$ were to rotate faster, accretion to the surface would become impossible due to the centrifugal barrier in the co-rotating magnetosphere.

Now consider the transfer of angular momentum near the accreting object. Infalling matter, as it reaches the magnetosphere, is accelerated until is co-rotates. This process 
removes angular momentum from the spinning neutron star. If the matter then falls to the surface, the angular momentum is replaced and no spin-down results; but if the rotation is so fast that complete accretion is prevented by centrifugal forces, then this form of drag serves to slow the rotation. The torque may be roughly $B_{m}^{2} R_{m}^{2}=$ $=B_{0}^{2} R_{0}^{6} R_{m}^{-3}$, which is larger than the vacuum pulsar-radiation torque by a factor of the order of $\left(R_{c} / R_{m}\right)^{3}$. This would be sufficient to appreciably alter the rotation of Cen X-3 in only a few hundred years. Evidently, such braking provides a suitable mechanism for slowing the spin until $R_{m} \sim R_{\text {sync }}$.

Another mechanism prevents the rotation from decreasing beyond that point. The pattern of accretion shown in Figure 2 is unlikely to have perfect symmetry; since the density of the stellar wind decreases outward while its speed increases, we might expect more gas to be accreted on that side of the flow pattern which faces the larger star. Consequently, if all of the accreted material were to reach the surface of the neutron star without somehow losing angular momentum, it would exert a torque whose order of magnitude is $\left(\dot{M}_{\mathrm{accr}} v_{\mathrm{rel}} a_{\mathrm{accr}}^{2} / r\right)$. This would alter the spin of Cen X-3 in $10^{4}$ or $10^{5} \mathrm{yr}$.

Thus we are led to a picture in which the rotation rate of the neutron star is held near an equilibrium value, such that the centrifugal barrier acts as a valve regulating the flow of angular momentum to the surface. Hot gas may collect around the magnetopause, occasionally escaping to carry away excess angular momentum. The rotation rate is determined chiefly by the surface magnetic field and the accretion rate. In equilibrium the period $P \propto\left(\dot{M}_{\text {accr }}\right)^{3 / 7}$ may be expected to decrease as the accretion rate increases.

Actual production of the X-ray luminosity remains to be discussed. The efficiency of energy release mentioned in Section 1 probably involves two distinct stages: (1) infalling material releases its kinetic energy, about $\left(R_{G} / R_{0}\right)\left(\dot{M}_{\mathrm{accr}} c^{2} / 2\right) \approx 0.05 \dot{M}_{\mathrm{accr}} c^{2}$, as it strikes the surface; and (2) a comparable amount of energy is released as the neutron star adjusts internally to its increasing mass. The latter process is like an internal energy source, which probably serves to maintain an effective surface temperature near $8 \times 10^{6} \mathrm{~K}$ that emits $\sim 10^{37} \mathrm{erg} \mathrm{s}^{-1}$ in a mildly directional fashion. The immediate process, which occurs in a localized area of the surface, may account for the observed X-ray pulses. Figure 3 shows schematically how the infalling material must be funneled along the field lines toward the magnetic polar regions. It is easy to estimate the radius of each polar funnel at the surface, by tracing the critical field lines shown in Figure 3 (assuming an approximately-dipolar field); the result is nearly

$$
r_{\text {pole }} \approx R_{0}^{3 / 2} R_{m}^{-1 / 2} \approx 0.8 \mathrm{~km}
$$

The situation at the base of the funnel is shown in Figure 4. Since the electron density in the funnel approaches $10^{21} \mathrm{~cm}^{-3}$ near the surface of the neutron star, the gas is optically thick to ordinary electron scattering (though the strong magnetic field must cause the opacity and the behavior of the gas to be very complicated). Radiation pressure may cause the infalling matter to release its kinetic energy over a distance comparable to $r_{\text {pole. }}$. The radiation will diffuse out through a cylindrical surface with 
height $\approx$ radius $=r_{\text {pole }}$. Supposing this surface to radiate like a black body, its temperature $T_{x}$ may be found from

$$
2 \pi r_{\text {pole }}^{2} \sigma T_{x}^{4} \approx L_{x, \text { pole }} .
$$

If $r_{\text {pole }}=0.8 \mathrm{~km}$ and $L_{x}$, pole $=10^{36} \cdot{ }^{5} \mathrm{erg} \mathrm{s} s^{-1}$, we find $T_{x} \approx 3.4 \times 10^{7} \mathrm{~K}$, which is in excellent agreement with the observed spectrum (Giacconi et al., 1971) - although admittedly, we have neglected to correct for the $10 \%$ gravitational redshift!

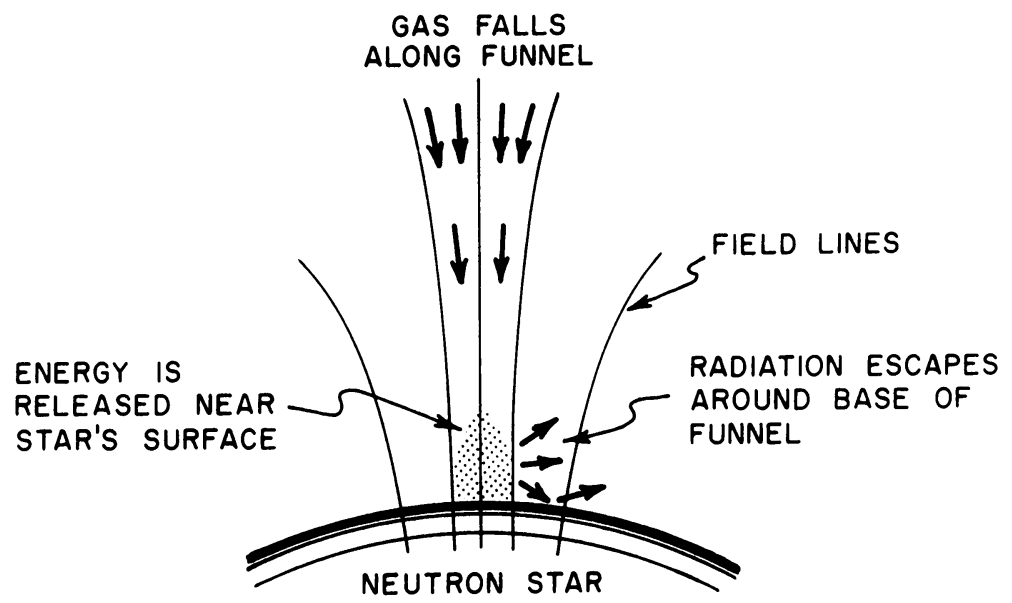

Fig. 4. Infalling material striking the surface near one magnetic pole of a neutron star.

As for the pulses, Figure 4 shows that the escaping radiation tends to move sideways relative to the funnel - favoring distant observers who are not far from the neutron star's magnetic-equatorial plane. However, since the emitting gas is moving downward at nearly a third the speed of light, there is a tendency for the radiation to move downward, to be reflected from the surface of the neutron star and then move upward in the form of a broad, hollow cone. If the neutron star has an extended 'atmosphere' of high-energy particles, refraction effects could narrow the cone. Hence, an observer may see pulses from the above model, and in any case will see a doubly periodic $\mathrm{X}$-ray luminosity as the two magnetic poles rotate through the line of sight.

\section{Conclusion}

This relatively simple stellar wind driven accretion model appears to satisfactorily account, in a quantitative but not precise way, for most of the observed features of Cen X-3; in particular the luminosity, spectrum, and period of the pulsed X-rays are calculated with reasonable accuracy. The non-pulsed X-ray component described by Tananbaum et al. (1972) is most likely radiation which has been scattered off electrons in the stellar wind, since the scattering optical depth outside the orbit of the neutron stars is of the order of 0.1 for the stellar wind described in Section 3. 
We have not explained the pulse shape in detail, nor do we propose a reason for the 'on' and 'off' modulation period of about thirty days (Tananbaum et al., 1972), which may have something to do with the outer layers of the massive star and its rate of mass ejection.

The total number of X-ray sources of the type discussed is small even if it is several times larger than discovered so far by the UHURU survey, due to radiation beaming effects, and the consequent total galactic $\mathrm{X}$-ray luminosity very small compared to $10^{10} L_{\odot}$ - the galactic luminosity. This new class of objects may prove very important, however, as astrophysical laboratories to study high density objects. In particular when the companion stars are discovered they will presumably also be found to be single line spectroscopic binaries with measurable mass functions. Then a determination of the inclination of the system (possible in principle by several means) will allow a direct experimental determination of a neutron star mass. If, further, we were lucky enough to discover a low mass third body orbiting the close binary as a distant visual companion, a direct test of the scaler tensor theory would be possible. This follows since Kepler's laws are not satisfied in the scalar tensor theory, the discrepancy for the neutron star being of order $R_{g} / R_{\odot} \sim 0.1$. The reader may invent at leisure other experiments possible in a system involving a clock in a deep potential well in nearly grazing orbit about a massive star.

\section{Acknowledgements}

The authors are pleased to acknowledge useful conversations with A. Hiltner, M. Rees, E. E. Salpeter, and many others. J.P.O. gratefully acknowledges the support he has received as an Alfred P. Sloan Fellow, 1970-72, and K.D. the support from National Aeronautics and Space Administration grant NGL-31-001-007.

\section{References}

Beals, C. S.: 1951, Publ. Dom. Astrophys. Obs. 9, 1.

Bolton, C.: 1971, Bull. Am. Astron. Soc. 3, No. 4.

Bondi, H. and Hoyle, F.: 1944, Monthly Notices Roy. Astron. Soc. 104, 273.

Carruthers, G. R.: 1968, Astrophys. J. 151, 269.

Eddington, A.: 1926, The Internal Constitution of the Stars, (reprinted by Dover, New York, 1959).

Gott, J. R., Gunn, J. E., and Ostriker, J. P.: 1970, Astrophys. J. 160, L91.

Gribben, J.: 1971, Nature 233, 19.

Hiltner, W. A., Werner, J., and Osmer, P.: 1972, Astrophys. J. Letters, (in press).

Jeans, J. H.: 1919, Problems of Cosmogony and Stellar Dynamics, Cambridge Univ. Press.

Jeans, J. H.: 1928, Astronomy and Cosmogony, Cambridge Univ. Press.

Leach, R. and Ruffini, R.: 1972, (preprint).

Lucy, L. B. and Solomon, P. M.: 1970, Astrophys. J. 159, 879.

Morton, D. C.: 1967, Astrophys. J. 147, 1017.

Morton, D. C.: 1969, Astron. Space Sci. 3, 117.

Ostriker, J. P.: 1968, Nature 217, 1227.

Ostriker, J. P., Rees, M., and Silk, J.: 1970, Astrophys. Letters 6, 179.

Ostriker, J. P. and Tassoul, J.-L.: 1968, Nature 219, 577.

Salpeter, E. E.: 1972, this volume, p. 135.

Schreier, E., Levinson, R., Gursky, H., Kellogg, E., Tananbaum, H., and Giacconi, R.: 1972, Astrophys. J. 172, L79. 
Tanabaum, H., Gursky, H., Kellogg, E. M., Levinson, R., Schreier, E., and Giacconi, R.: 1972, Astrophys. J. Letters, (in press).

van den Heuvel, E. P. J.: 1970, in A. Slettebak (ed.), Stellar Rotation, D. Reidel Publ. Co., DordrechtHolland, p. 178.

van den Heuvel, E. P. J. and Heise, J.: 1972, Nature, (in press).

Webster, B. L. and Murdin, P.: 1972, Nature 235, 37.

Wilson, R. E.: 1972, Astrophys. J. 174, L27.

Zel'dovich, Ya. B. and Novikov, I. D.: 1964, Sov. Phys. Dokl. 9, 246.

Zel'dovich, Ya. B. and Novikov, I. D.: 1971, Relativistic Astrophysics, Vol. 1, Univ. of Chicago Press, Chicago, Chap. 13. 